\title{
Idiopathic Granulomatous Mastitis- Challenges of Diagnosis and Treatment
}

\author{
Roy $\mathrm{S}^{1}$, Mondal $\mathrm{SK}^{2}$, Amin $\mathrm{R}^{3}$, Khan $\mathrm{IS}^{4}$
}

\begin{abstract}
Idiopathic Granulomatous Mastitis (IGM) is a benign breast disease. Owing to its unknown aetiology, rareness, variation of presentation - diagnosis and treatment is still a challenge. This is a retrospective review of clinical presentation and investigation finding of all patients histopathologically diagnosed as IGM from February 2010 to January 2014. Treatment outcome and follow up information were recorded. $6(42 \%)$ patient presented with breast abscess, $5(35.7 \%)$ patient had features of mastitis without abscess, $3(21.4 \%)$ patient had painful firm to hard lump. Diagnosis confirmed by excisional/ incisional biopsy. $9(64 \%)$ patients needed more than one surgical intervention. Mean follow up period was 27 months. Standardization and optimization of treatment is yet to be achieved.
\end{abstract}

Key Words: Idiopathic granulomatous mastitis, Benign, Abscess, Excisional biopsy, Retrospective review.

1. Corresponding Author: Dr. Sharmistha Roy Assistant Professor, Department of Surgery BIRDEM General Hospital \&IMC

2. Dr. Samiron Kumar Mondal Associate Professor, Department of Surgery BIRDEM General Hospital \&IMC

3. Dr. Md. Ruhul Amin Professor \& Head of Department, Department of Physiology Sylhet M A G Osmani Medical College

4. Dr. Indira Sufia Khan Associate Professor, Department of Physiology Medical College for Women \& Hospital

\section{Introduction}

Idiopathic Granulomatous Mastitis(IGM) is a rare chronic inflammatory breast disease. IGM was first described by Kessler and Wolloch in $1972^{1}$. Because of its rarity, unknown etiology and notoriously recurrent characteristics diagnosis and treatment is still a challenge. IGM is important for two reason, one -it mimics carcinoma breast in clinical presentation and imaging and two-it is difficult to treat specially when presented with recurrent abscess and fistula formation. The aim of this study was to describe the clinical signs, radiological findings, management, clinical course and the recurrence rate of the patients who were treated for to IGM.

\section{Materials and Methods}

Data regarding clinical features at first presentation, imaging reports were collected from all patients who were diagnosed as Idiopathic Granulomatous Mastitis by cytology or histopathology. Treatment was started as per current consensus of treatment strategy and these patients were followed up care fully for at least 1 year to see the course of disease and recurrence. History taken from patient regarding Age, pregnancy, lactation, use of contraceptives, patient diabetic or not. All patient underwent physical examinationand an ultra sonogram. In Patient over 40 years mammogram also done. One patient with multiple discharging sinus underwent MRI.

Histopathological diagnosis obtained by wide local excision with a $1 \mathrm{~cm}$ disease free margin in patients presented with lump and FNAC/ Core Biopsy suggesting IGM. Incision and drainage of pus with biopsy from wall of abscess was taken in patient presenting with abscess formation. Pus was also sent for AFB staining, Culture sensitivity and presence of atypical mycobacteria, Nocardia, Or Chlamydial infection were also ruled out.Tissue from sinus tract were also sent for histopathology in one patient who presented with multiple discharging sinus, 6 month after initial treatment for abscess formation.

Histopathology showed chronic inflammatory reaction with granulomas which composed of epitheloidhistiocytes, Langhans giant cells accompanied by lymphocytes, plasma cells and occasional eosinophils and no caseation.

Physical examination and followup ultrasound was done in all patients monthly, until complete resolve. Patients were then under follow up with history \& physical examination upto 1 year of complete resolve to see recurrence.

\section{Result}

During our study period February 2010 to January 2014 a total of 4879 patients attended surgical OPD. Among them 352 were diagnosed initially as mastitis or breast abscess. Cytology or core biopsy was sent for 176 patient. Among them 2 had diagnosis of carcinoma breast, 21 had granulomatous mastitis, and the rest had nonspecific 
mastitis, galactocoele, ductectasia. These 21 cases, pathological diagnosis was reconfirmed by excisional biopsy, core biopsy or incision and drainage with biopsy from wall of abscess cavity. After excluding AFB for TB, atypical mycobacteria, culture sensitivity, chlamydial or nocardia infection 11 patient were finally diagnose as Idiopathic Granulomatous Mastitis. 411 patient initially diagnosed as suspicious breast lump ie malignancy were evaluated and 3 of them ultimately came out as IGM. So our total number of case with confirmed diagnosis of IGM in a period of 4 years were 14(n).

Patient Profile: Symptoms of these 14 patients at initial presentation, examination findings, and imaging were then reviewed. Mean age of patients were 41(23-57). 9 were diabetic, 5 non diabetic. All our patients were married, 5 nalipara. 2 of the ladies were lactating mother, with age of last child less than one year. 4 of them has taken contraceptives for more than 2 years.

Presentation: On physical examination 6(42\%)patient had signs of inflammation with fluctuating area of abscess formation at presentation. 5(35.7\%)patient had a inflamed indurated area but no evidence of abscess clinically and on imaging. 3(21.4\%)patient had no features of inflammation but a painful firm to hard lump.

Diagnosis: Ultrasonography (USG) was performed for all of the patients. USG showed an irregular heterogeneous mass in all cases, and a hypo echoic abscess in 7 cases. 5 patients aged over 40 years were examined with mammography. An ill-defined tumor was observed in 3 patients, and diffusely increased asymmetric density was observed in 2 patients. MRI was done in one patient who developed multiple discharging sinus, 6 months after initial drainage of abscess.

Patients without frank abscess(total 8) were sent for FNAC. When granulomatous mastitis was suggested report was reconfirmed by core biopsy in 5 patient (with features of mastitis) and excision biopsy in 3 patient (with hard lump).

Treatment: 6 patient with breast abscess were treated with Incision and drainage (I\&D). Biopsy sent from tissue in abscess cavity wall confirmed diagnosis of IGM. After regular dressing secondary closure of the wound was done. 5 Patients with mastitis without pus formation were treated with empherical antibiotics for at least 2 weeks. 2 resolved and 3 developed abscess, requiring I\&D. Lumpectomy with $1 \mathrm{~cm}$ healthy margin was done in 3 patients. All diabetic patients were shifted to insulin from oral anti diabetic drugs before surgery to achieve a better glycemic control.

Follow up: Follow up was done for at least 1 year after complete resolve of disease. Mean follow up was 27 months(14-56 months). Physical examination and USG was done in monthly follow up. 2 cases(14\%) had no recurrence after incision drainage of abscess, followed by dressing and secondary closure. 3(21.4\%) patient developed recurrent abscess more than once, requiring repeat surgeries. One patient developed discharging sinus(7\%), and wound was kept open after excision of sinus tracts, allowing to heal with regular dressing. 3 patient out of 5 treated with antibiotics initially, developed abscess later, after stopping antibiotic. They were treated with I\&D. Among 3 lumpectomy patients 2 developed hypoechoic collection on F/U USG. USG guided aspiration was done and sent for $\mathrm{c} / \mathrm{s}$. One of these patient later developed abscess inspite of using antibiotics and required I\&D.

\section{Discussion}

Idiopathic Granulomatous Mastitis is a rare benign disease of breast affecting mostly reproductive age ladies ${ }^{2}$. In our series all but one of our patients were in reproductive age. Many causative agents have been reported to be linked with the condition like - local irritant, oral contraceptive pill, viruses, mycotic and parasitic infections, hyperprolactinemia, diabetes mellitus, smoking, alfa 1 antitrypsin deficiency, autoimmunity have been proposed to explain the etiology of IGM but never proven. ${ }^{2-7}$

IGM presents most commonly with a unilateral painful, firm, tender, ill defined mass in the breast ${ }^{7,8}$. The lesions may be located in any quadrant of the breast ${ }^{7}$. In consequence of granulomatous inflammation, IGM can cause skin thickness, sinus and abscess formation, axillary lymphadenopathy and nipple retraction. Which may be clinically mistaken for breast carcinoma ${ }^{2,7,8}$. In our series 3 out of 14 patient was clinically thought to be malignant due to their presentation.

The information, obtained from US and MMG is nonspecific and lack of specificity to diagnose IGM or to exclude breast carcinoma. Memis et al reported the appearance of irregular hypoechoic mass lesions and tubular hypoechoic areas connecting to the mass as frequent sonographic features ${ }^{9}$. In other studies, parenchymal heterogeneity and areas of mixed echo pattern have been reported ${ }^{6}$. Distinct from these lesions enlarged axillary lymph nodes give rise to thought a locally advanced breast carcinoma ${ }^{6}$. In our series USG showed an irregular heterogeneous mass in all cases, and a hypo echoic abscess in 7 cases. In our experience when physical examination mimics features of malignancy, USG finding of heterogenousecogenicity with hypoechoic collection suggests presence of an inflammatory process. On mammograman ill defined mass, asymmetrically increased diffuse or focal density without parenchymal distortion or microcalcification are the most common finding ${ }^{6,10}$. In our series we did mammogram in 5 patients who were above 40 years. An ill-defined tumor was observed in 3 patients, and diffusely increased asymmetric density was observed in 2 patients. No micro calcification was seen. According to some studies, MRI does not provide adjunct information for the differentiation of IGM from carcinoma ${ }^{6}$. 
But Dursun et al. reported that the kinetic features are usually nonsuspicious and can be helpful in the differential diagnosis from carcinoma ${ }^{10}$. Others consider MRI does not play a role in the differential diagnosis between other inflammatory and granulomatous diseases and IGM ${ }^{7,8,10}$. We did MRI in only one patient, not as a diagnostic tool but to delineate sinus tracts, and their retromammary extension.

Histopathologic diagnosis can be achieved with fine needle aspiration cytology (FNAC), core biopsy, incisional or excisional biopsy ${ }^{6}$. But the usefulness and reliability of FNAC has been debated, because various causes of granulomatous inflammation cannot be differentiated with $\mathrm{FNAC}^{5,11,12}$. In our patients, the histopathologic diagnosis was obtained from excisional and incisional biopsies, we have not prefferred FNAC.

There is still no commonly accepted treatment for IGM. We consider that wide local excision is the better treatment choice if possible. But, complicated IGM with abscess, fistula and diffuse involvement is difficult to treat $\&$ has a high recurrence rate. The optimal treatment of IGM remains controversial. Surgical excision still seems to be the best treatment. Wide local excision can be appropriate treatment also provide exact diagnosis. After wide local excision, usually further therapy is not needed. Different recurrence rates (range 5.5\%-50\%) are reported after wide local excision ${ }^{7,8}$. There was 1 recurrence of 3 patients that wide local exsicion was performed in our patients. Our recurrence rate is $33.3 \%$. In patients with abscess we had 4 recurrences out of 6 incision and drainage $(66 \%)$. The possible cause of higher recurrence in our series may be diabetes mellitus. In our series 9 out of 14 patient were diabetic. All diabetic patients were shifted to insulin from oral anti diabetic drugs before surgery to achieve a better glycemic control.

In conclusion, Idiopathic Granulomatous Mastitis is a diagnosis of exclusion. This rare inflammatory condition of breast is difficult to distinguish from other inflammatory condition or carcinoma of breast clinically or through imaging. Treatment is challenging both from patient side and surgeon's side. A very informative counseling at the very beginning will help the patient to cope with the nature and course of disease and treatment. The final cosmetic outcome may be a cause of concern in complicated cases. Patience, perseverance, adequate and timely surgery with the adjunct of medical treatment should be the key to success.

\section{Referrences}

1. Kessler E, Wolloch Y. Granulomatous mastitis: a lesion clinically simulating carcinoma. Am J Clin Pathol.1972;58(6):642-6.

2. Ozel L, Unal A, Unal E, Kara M, Erdogdu E, Krand O, et al. Titiz MI: Granulomatous mastitis: is it autoimmune disease? diagnostic and therapeutic dilemmas. Surg Today. 2012;42(8):729-33.

3. Akbulut S, Arikanoglu Z, Senol A, Sogutcu N, Basbug $\mathrm{M}$, Yeniaras E, et al. Is methotrexate an acceptable treatment in the management of idiopathic granulomatous mastitis? Arch Gynecol Obstet.2011;284(5):1189-95.

4. Vinayagam R, Cox J, Webb L. Granulomatous mastitis: a spectrum of disease. Breast Care. 2009;4(4):251-4.

5. Bakaris S, Yuksel M, Çiragil P, Atahan Güven M, Ezberci F, Bulbuloglu E. Granulomatous mastitis including breast tuberculosis and idiopathic lobular granulomatous mastitis. Can J Surg.2006;49(6):427-30.

6. Gurleyik G, Aktekin A, Aker F, Karagulle H, Saglam A.Medical and surgical treatment of idiopathic granulomatous lobular mastitis: a benign inflammatory disease mimicking invasive carcinoma. J Breast Cancer.2012;15(1):119-23.

7. Patel RA, Strickland P, Sankara IR, Pinkston G, Many W, Rodriguez M. Idiopathic granulomatous mastitis: case reports and review of literature. J Gen Intern Med.2009;25 (3): 270-3.

8. Akcan A, Akyildiz H, Deneme MA, Akgun H, Aritas Y. Granulomatous lobular mastitis: a complex diagnostic and therapeutic problem. World J Surg.2006;30:1403-9.

9. Memis A, Bilgen I, Ustun EE, Ozdemir N, Erhan Y, Kapkaç M. Granulomatous mastitis: imaging findings with histopathologic correlaton. Clin Radiol. 2002;57:1001-6.

10. Dursun M, Yilmaz S, Salmaslioglu A, Yavuz E, Igci A, Acunas G, et al. Multimodality imaging features of idiopathic granulomatous mastitis: outcome of 12 years of experience. Radiol Med.2012;117(4):529-38.

11. Tse GMK, Poon CSP, Law BKB, Pabg LM, Chu WCW, Ma TKF. Fine needle aspiration cytology of granulomatous mastitis. J Clin Pathol.2003;56:519-21.

12. Lacambra M, Thai TA, Lam CCF, Yu AMC, Pham HT, Tran PVT, et al. Granulomatous mastitis: the histological differentials. J Clin Pathol. 2011;64: 405-11. 